\title{
MAGNETO-HYDRO DYNAMIC FLOW AND HEAT TRANSFER OF NON- NEWTONIAN POWER-LAW FLUID OVER A NON-LINEAR STRETCHING SURFACE WITH VISCOUS DISSIPATION
}

\author{
N. KISHAN \\ Department of Mathematics, Osmania University \\ Hyderabad -7, A.P., INDIA \\ E-mail: kishan_n@rediffmail.com \\ P. KAVITHA* \\ Nalla Narashima Reddy Education \\ Society's Group of Institutions \\ Ghatkesar, RR-88, A.P., INDIA \\ E-mail: pagdikavitha@gmail.com
}

\begin{abstract}
A fluid flow and heat transfer analysis of an electrically conducting non-Newtonian power law fluid flowing over a non-linear stretching surface in the presence of a transverse magnetic field taking into consideration viscous dissipation effects is investigated. The stretching velocity, the temperature and the transverse magnetic field are assumed to vary in a power-law with the distance from the origin. The flow is induced due to an infinite elastic sheet which is stretched in its own plane. The governing equations are reduced to non-linear ordinary differential equations by means of similarity transformations. By using quasi-linearization techniques first linearize the non linear momentum equation is linearized and then the coupled ordinary differential equations are solved numerically by an implicit finite difference scheme. The numerical solution is found to be dependent on several governing parameters, including the magnetic field parameter, power-law index, Eckert number, velocity exponent parameter, temperature exponent parameter, modified Prandtl number and heat source/sink parameter. A systematic study is carried out to illustrate the effects of these parameters on the fluid velocity and the temperature distribution in the boundary layer. The results for the local skin-friction coefficient and the local Nusselt number are tabulated and discussed.
\end{abstract}

Key words: magneto-hydrodynamic flow, power-law fluid, stretching sheet, heat source/sink parameter, viscous dissipation.

\section{Introduction}

A number of industrially important fluids such as molten plastic, polymers, and pulp foods exhibit non-Newtonian fluid behavior. A non-Newtonian fluid is a fluid in which the viscosity changes with the applied strain rate. As a result, non-Newtonian fluids may not have well defined viscosity. In modern technology and in industrial applications, non-Newtonian fluids play an important role. Many modern materials and manufacturing processes involve non-Newtonian fluids. Examples of non-Newtonian behavior can be found in processes for manufacturing coated sheets, optical fibers, foods, drilling mud's and plastics polymer. For a non-Newtonian fluid, the viscous stress is a nonlinear function of the rate of deformation, and the relationship for the power-law fluid is given by

\footnotetext{
* To whom correspondence should be addressed
} 


$$
\tau_{x y}=\mu\left|\frac{\partial u}{\partial y}\right|^{n-1} \frac{\partial u}{\partial y}
$$

where $\tau_{x y}$ is the shear stress, $\mu$ is the consistency index and $n$ is the power-law index. When $n<1$, the fluid is described as pseudo-plastic, $n>1$ dilatant and when $n=1$, it is a Newtonian fluid. Several studies in the literature suggest the range $0<n \leq 2$ for the power-law index $n$. Schowalter (1960) was the first one, who formulated the boundary layer flow of a non-Newtonian fluid and established the conditions for the existence of a similarity solution. Acrivous et al. (1960) considered the problem of natural convection heat transfer to power-law fluids for different geometric configurations. Zheng et al. (2000) investigated the flow in powerlaw fluid over a flat plate moving at constant speed in the direction and opposite to the direction of the main stream. The flow of an incompressible viscous fluid and heat transfer phenomena over a stretching sheet have received great attention during the last decades owing to the abundance of practical applications in chemical and manufacturing processes, such as polymer extrusion, drawing of copper wires, continuous casting of metals, wire drawing and glass blowing. Since the pioneering work of Sakiadis (1961), various aspects of the problem have been investigated by many authors. The various aspects of the stretching sheet problem involving Newtonian/non-Newtonian fluids have been extensively studied by Crane (1970), Gupta and Gupta (1977), Jadhav and Waghmode (1990), Rajagopal et al. (1983), Chen (2003). These research works do not however consider the situation where hydro-magnetic effects arise. The study of hydrodynamic flow and heat transfer over a stretching sheet may find its applications in polymer technology related to the stretching of plastic sheets. Also, many metallurgical processes involve the cooling of continuous strips or filaments by drawing them through a quiescent fluid and while drawing these strips are sometimes stretched. The rate of cooling can be controlled by drawing such strips in an electrically conducting fluid subjected to a magnetic field in order to get the final products of desired characteristics; as such a process greatly depends on the rate of cooling. In view of this, the study of an MHD flow of Newtonian/non-Newtonian flow over a stretching sheet was carried out by many researchers (Sarpakaya, 1961; Andersson et al., 1992, Char, 1994; Cortell, 2005).

The problem in particular has applications in polymer industry in which most of the fluids used are known not to obey the usual linear relationship between stresses and the rate of strain, which is assumed for Newtonian fluids.

For the non-Newtonian power-law fluids, the hydro-magnetic problem of the MHD boundary layer flow over a continuously moving surface over a stretching sheet has been dealt with by several authors, e.g., Mahmoud and Mahmoud (2006). The effect of the magnetic field is found to decrease the velocity distribution and thus to increase the skin-friction coefficient. However, relatively less attention has been paid to the accompanying heat transfer problem of power-law fluids past a stretching surface in the presence of a magnetic field. The MHD free-convection flow of a non-Newtonian power-law fluid at a stretching surface with a uniform fee-stream was studied by Emad et al. (2005).

All the above mentioned investigators confined their analyses to on MHD flow and heat transfer over a linear stretching sheet. However, the intricate flow and heat transfer problem over a non-linearly stretching sheet with the effects of internal heat generation/absorption is yet to be studied. This has applications to several industrial problems such as engineering processes involving nuclear power plants, gas turbines and many others and has been studied by Chaim (1995), Ishak et al. (2006), Anjali Devi and Thiyagarajan (2006). The main concern of the present paper is to study the effect of variable thermal conductivity on the power-law fluid flow and heat transfer over a non-linearly stretching sheet in the presence of a transverse magnetic field by taking into account viscous dissipation effects. Because of the intricacy, the influence of the power-law index parameter, magnetic parameter, non-linear velocity and temperature exponent and heat source/sink parameter make the momentum and energy equations coupled and highly non-linear partial differential equations. To reduce the number of independent variables, these partial differential equations are simplified to couple non-linear ordinary differential equations by suitable similarity transformations. The non-linear differential equations are linearized by using Quasi-linearization technique. These equations are in 
turn solved numerically by an implicit finite-difference scheme along with the Gauss Seidal method with the help of C-programming.

\section{Mathematical formulation}

Consider a steady laminar two-dimensional boundary layer flow of a viscous incompressible and electrically conducting fluid obeying the power-law model in the presence of a transverse magnetic field $B_{0}$. The flow is generated as a consequence of non-linear stretching of the boundary sheet, caused by a simultaneous application of two equal and opposite forces along the $x$-axis, while keeping the origin fixed in the fluid of the ambient temperature $T_{\infty}$. The momentum and energy equations for a fluid with variable thermal conductivities and variable viscosities in the presence of an eternal source/sink, viscous dissipation are studied. The positive $x$-coordinate is measured along the direction of the motion, with the slot at the origin, and the positive $y$-coordinate is measured normal to the surface of the sheet and is positive from the sheet to the fluid. The continuous stretching sheet is assumed to have a non-linear velocity and prescribed temperature of the form $U(x)=b x^{m}$ and $T_{w}(x)=T_{\infty}+A x^{r}$, respectively, where $b$ is the stretching constant, $x$ is the distance from the slot; $A$ is a constant whose value depends upon the properties of the fluid. Here, $m$ and $r$ are the velocity and temperature exponents, respectively. It is also assumed that the magnetic Reynolds number $\mathrm{Re}_{m}$ is very small; i.e., $\mathrm{Re}_{m}=\mu_{0} \sigma b l<<1$ where $\mu_{0}$ the magnetic permeability and $\sigma$ is the electric conductivity. We neglect the induced magnetic field, which is small in comparison with the applied magnetic field. Further, the external electrical field is assumed to be zero and the electric field due to polarization of charges is also negligible. Under these assumptions, the basic equations governing the flow and heat transfer in usual notation are

$$
\begin{aligned}
& \frac{\partial u}{\partial x}+\frac{\partial v}{\partial y}=0, \\
& u \frac{\partial u}{\partial x}+v \frac{\partial u}{\partial y}=-v \frac{\partial}{\partial y}\left(-\frac{\partial u}{\partial y}\right)^{n}-\frac{\sigma B_{0}}{\rho} u \\
& u \frac{\partial T}{\partial x}+v \frac{\partial T}{\partial y}=\alpha \frac{\partial^{2} T}{\partial y^{2}}+\frac{Q_{s}}{\rho c_{p}}\left(T-T_{\infty}\right)+\mu\left(\frac{\partial u}{\partial y}\right)^{2}
\end{aligned}
$$

where $u$ and $v$ are the flow velocity components along the $x$ and $y$-axes, respectively, $v$ is the kinematic viscosity of the fluid, $n$ is the power-law index, $\rho$ is the fluid density and $c_{p}$ is the specific heat at constant pressure. The first term in the right hand side of Eq.(2.2) is the shear rate $\left(\frac{\partial u}{\partial y}\right)$. It has been assumed to be negative throughout the boundary layer since the stream wise velocity component $u$ decreases monotonically with the distance $y$ from the moving surface (for continuous stretching surface). A rigorous derivation and subsequent analysis of the boundary layer equations, for power-law fluids, were recently provided by Denier and Dąbrowski (2004). They focused on a boundary layer flow driven by a free stream $U(x) \approx x^{m}$ i.e., of Falkner-Skan type. Such boundary layer flows are driven by a stream wise pressure gradient $-\frac{d p}{d x}=\rho \frac{d u}{d x}$ set up by the external free stream outside the viscous boundary layer. In the present context no driving pressure gradient is present. Instead the flow is driven solely by the stretching surface, which moves with a prescribed velocity. $T$ is the temperature of the fluid and $U(x) . T$ is the temperature of 
the fluid and $\alpha$ is the thermal diffusivity of the fluid. The last term containing $Q_{s}$ in Eq.(2.3) represents the temperature-dependent volumetric rate of the heat source when $Q_{s}>0$ and heat sink when $Q_{s}<0$. These deal with the situation of exothermic and endothermic chemical reactions respectively. Thus the relevant boundary conditions applicable to the flow are

$$
\begin{aligned}
& u(x, 0)=U(x), \\
& v(x, 0)=0, \\
& T(x, 0)=T_{w}(x), \\
& u(x, y) \rightarrow 0, \quad T(x, y) \rightarrow T_{\infty}, \quad \text { as } \quad y \rightarrow \infty .
\end{aligned}
$$

Here, boundary condition (2.7) means that the stream wise velocity and the temperature vanish outside the boundary layer. Equation (2.6) is the variable prescribed surface temperature at the wall, whereas Eq.(2.5) signifies the importance of impermeability of the stretching surface and Eq.(2.4) assures no slip at the surface. In order to obtain the similarity solutions of Eqs (2.1)-(2.7), we assume that the variable magnetic field $B_{0}(x)$ is of the form $B_{0}(x)=B_{0} x^{(m-1)} / 2$. This form of $B_{0}(x)$ has also been considered in the studies of MHD flow problems past moving or fixed flat plate. The momentum and energy equations can be transformed to the corresponding ordinary differential equations by the following transformation

$$
\begin{aligned}
& \eta=\frac{y}{x}\left(\operatorname{Re}_{x}\right)^{\frac{1}{n+1}}, \quad \theta(\eta)=\frac{T-T_{\infty}}{T_{w}-T_{\infty}}, \\
& \psi(x, y)=U x\left(\operatorname{Re}_{x}\right)^{\frac{-1}{n+1}} f(\eta)
\end{aligned}
$$

where $\eta$ is the similarity variable, $\psi(x, y)$ is the stream function $f$ and $\theta$ are the dimensionless similarity function and temperature, respectively. The velocity $u$ components and $v$ are given by

$$
u=\frac{\partial \psi}{\partial y}, \quad v=-\frac{\partial \psi}{\partial x} .
$$

The local Reynolds number is defined by

$$
\operatorname{Re}_{x}=\frac{U^{2-n} x^{n}}{v}
$$

The mass conservation Eq.(2.1) is automatically satisfied by Eq.(2.9). By assuming the similarity function $f(\eta)$ to depend on the similarity variable $\eta$, the momentum Eq.(2.2) and the heat Eq.(2.3) transform into the coupled non-linear ordinary differential equations of the form

$$
n\left(-f^{\prime \prime}\right)^{n-1} f^{\prime \prime \prime}-m f^{\prime 2}+\left(\frac{2 m n-m+1}{n+1}\right) f f^{\prime \prime}-M f^{\prime}=0
$$




$$
\theta^{\prime \prime}+\operatorname{Pr}\left(\frac{2 m n-m+1}{n+1}\right) f \theta^{\prime}+\operatorname{Pr}\left(S \theta-r f \theta^{\prime}\right)-\operatorname{Ec}\left(f^{\prime \prime}\right)^{2}=0
$$

The boundary conditions Eqs (2.4)-(2.7) now become

$$
\begin{array}{ll}
f(\eta)=0, & f^{\prime}(\eta)=1, \quad \theta(\eta)=1, \quad \text { at } \quad \eta=0, \\
f^{\prime}(\eta) \rightarrow 0, & \theta(\eta) \rightarrow 0, \quad \text { as } \quad \eta \rightarrow \infty
\end{array}
$$

where $M=\frac{\sigma B_{0}^{2}}{\rho b}$ is the magnetic parameter, $\operatorname{Pr}=\frac{\mathrm{Npe}_{x}}{\left(\mathrm{Re}_{x}\right)^{\frac{2}{n+1}}}$ is the modified Prandtl number for power-law fluids, $\mathrm{Npe}_{x}=\frac{c_{p} \rho U x}{k}$ is the convectional Peclet number (Andersson et al., 1992), $S=\frac{Q_{s}}{\rho c_{p} b}$ is the heat source/sink parameter, $\mathrm{Ec}=\frac{\rho c}{k} \mu U^{2}$ is the local Eckert number. Here, primes and the subscript $\eta$ denote differentiation with respect to $\eta$. Equations (2.11) and (2.12) are solved numerically subject to the boundary conditions Eq.(2.13). It should be noted that the velocity $U=U(x)$ is used to define the dimensionless stream function in Eq.(2.11). The local Reynolds number in Eq.(2.10) describes the velocity of the moving surface that drives the flow. This choice contrasts with the conventional boundary layer analysis, in which the free stream velocity is taken as the velocity scale. Although the transformation defined in Eqs (2.8) and (2.10) can be used for arbitrary variation of the transformation results in a true similarity problem only if $U$ varies as $b x^{m}$. Such surface velocity variations are therefore required for the ordinary differential Eq.(2.11) to be valid. The physical quantities of interest are the skin-friction coefficient $C_{f}$ and the local Nusselt number $\mathrm{Nu}_{x}$, which are defined as

$$
C_{f}=\frac{2 \tau_{w}}{\rho U^{2}}, \quad \quad \mathrm{Nu}_{x}=\frac{x q_{w}}{k\left(T_{w}-T_{\infty}\right)},
$$

respectively, where the wall shear stress $\tau_{w}$ and heat transfer from the sheet $q_{w}$ are given by

$$
\tau_{w}=\mu_{0}\left(\frac{\partial u}{\partial y}\right)_{y=0}, \quad q_{w}=-k\left(\frac{\partial T}{\partial y}\right)_{y=0},
$$

with $\mu_{0}$ and $k$ being the dynamic viscosity and thermal conductivity, respectively. Using the nondimensional variables (2.7), we obtain

$$
\begin{aligned}
& C_{f}=\left(\frac{-2 \tau_{x y}}{\rho(b x)^{2}}\right)_{y=0}=2\left[-f^{\prime \prime}(0)\right]^{n}\left[\mathrm{Re}_{x}\right]^{\frac{-1}{n+1}}, \\
& \mathrm{Nu}_{x}=-\left[\operatorname{Re}_{x}\right]^{\frac{1}{n+1}} \theta^{\prime}(0)
\end{aligned}
$$

where $\tau_{x y}$ is the shear stress and $\mathrm{Re}_{x}$ is the local Reynolds number. 


\section{Numerical procedure}

The system of transformed governing non-linear coupled differential Eqs (2.11) and (2.12) with the boundary conditions (2.13) is solved numerically using the finite-difference scheme. This method is unconditionally stable and has second-order accuracy with arbitrary spacing. The transformed non-linear differential equations are first linearized by Quasi-Linearization technique discussed by Bellman and Kalaba (1965). For the sake of brevity, the details of the numerical solution procedure are not presented here. It is worth mentioning that a uniform grid is satisfactory in obtaining sufficient accuracy with an error tolerance less than $10^{-5}$.

\section{Results and discussions}

The computations have been carried out for various values of the magnetic field parameter $M$, power-law index $n$, Eckert number Ec, velocity exponent parameter $m$, temperature exponent parameter $r$, modified Prandtl number Pr and heat source/sink parameter $S$. Numerical computations of the problem are performed by the implicit finite difference scheme along with the Gauss-Seidal iteration method with the help of C-programming.

Figures 1a-1c represent the horizontal velocity profile $f^{\prime}$ with $\eta$ for different values of the velocity exponent parameter $m$ for a) pseudo plastic fluids $(n=0.8)$ b) Newtonian fluids $(n=1)$ c) dilatent fluids $(n=1.6)$. From the geometrical representation we observe that the effect of increasing values of the velocity exponent parameter $m$ is to reduce the momentum boundary layer thickness, which tends to zero as the space variable $\eta$ increases from the boundary surface. Physically, $m<0$ implies that the surface is decelerated from the slot, $m=0$ implies the continuous momentum of a flat surface and $m>0$ implies that the surface is accelerated from the extended slit. The velocity profile $f^{\prime}$ decreases with the increase of the stretching sheet parameter $m$.
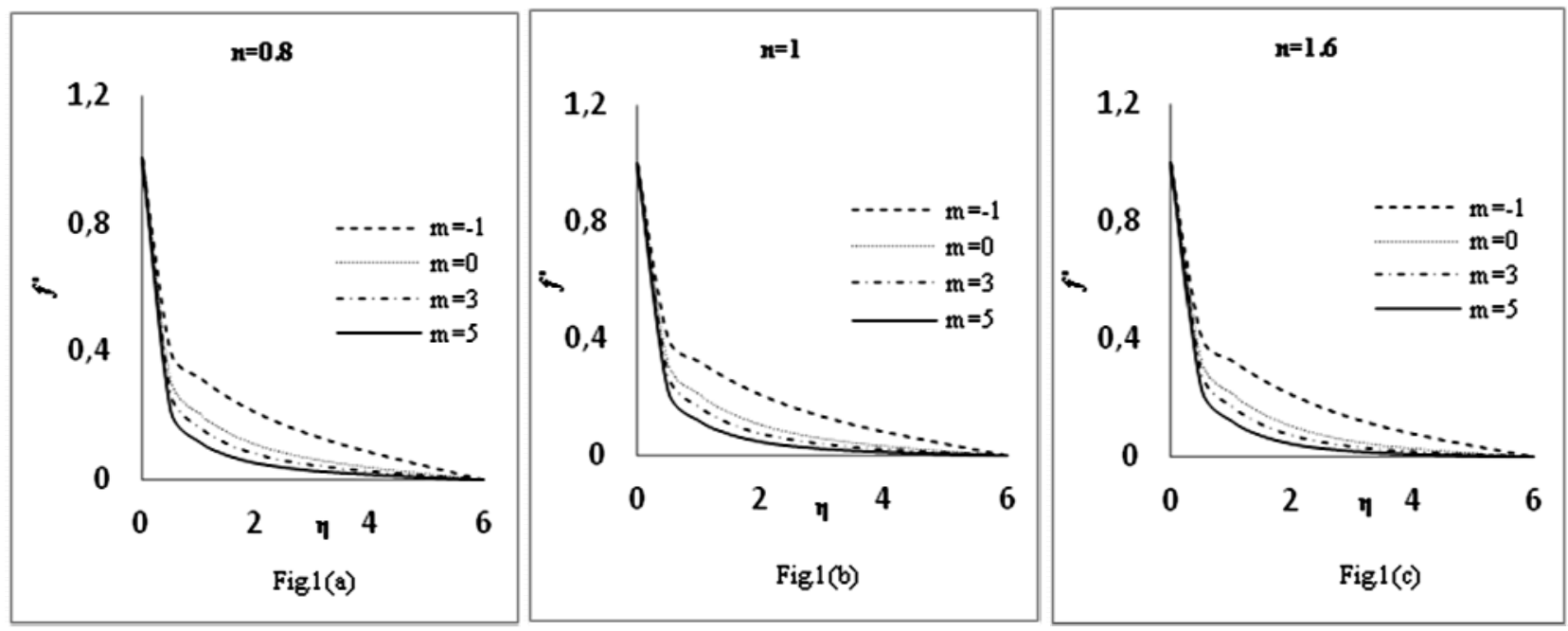

Fig.1. Velocity profiles for different values of $m$ for $M=0, \operatorname{Pr}=1, r=0, S=0, \mathrm{Ec}=0$ a) pseudo plastic fluids $(n=0.8)$ b) Newtonian fluids $(n=1)$ c) dilatent fluids $(n=1.6)$.

Figures 2a-2c demonstrate the dimensionless velocity profile $f^{\prime}$ for the different values of the magnetic field parameter $M$ for a) pseudo plastic fluids $(n<1)$ b) Newtonian fluids $(n=1)$ c) dilatent fluids $(n>1)$ respectively. The effect of the magnetic field parameter results in flattering of velocity profile $f^{\prime}$ in all the cases. The graphs of temperature profiles $\theta$ for a) pseudo plastic fluids $(n<1)$ b) Newtonian fluids $(n=1)$ c) dilatent fluids $(n>1)$ for different values of the stretching sheet parameter $m$ in the presence/absence of a 
magnetic field parameter $M$ is shown in Figs 3 and 4, respectively. The effect of increasing values of the stretching sheet parameter $m$ is to decrease temperature profiles $\theta$. However, the effect of the stretching sheet parameter $m$ is larger in the presence of the magnetic field parameter when compared with the effect in the absence of the magnetic field parameter.
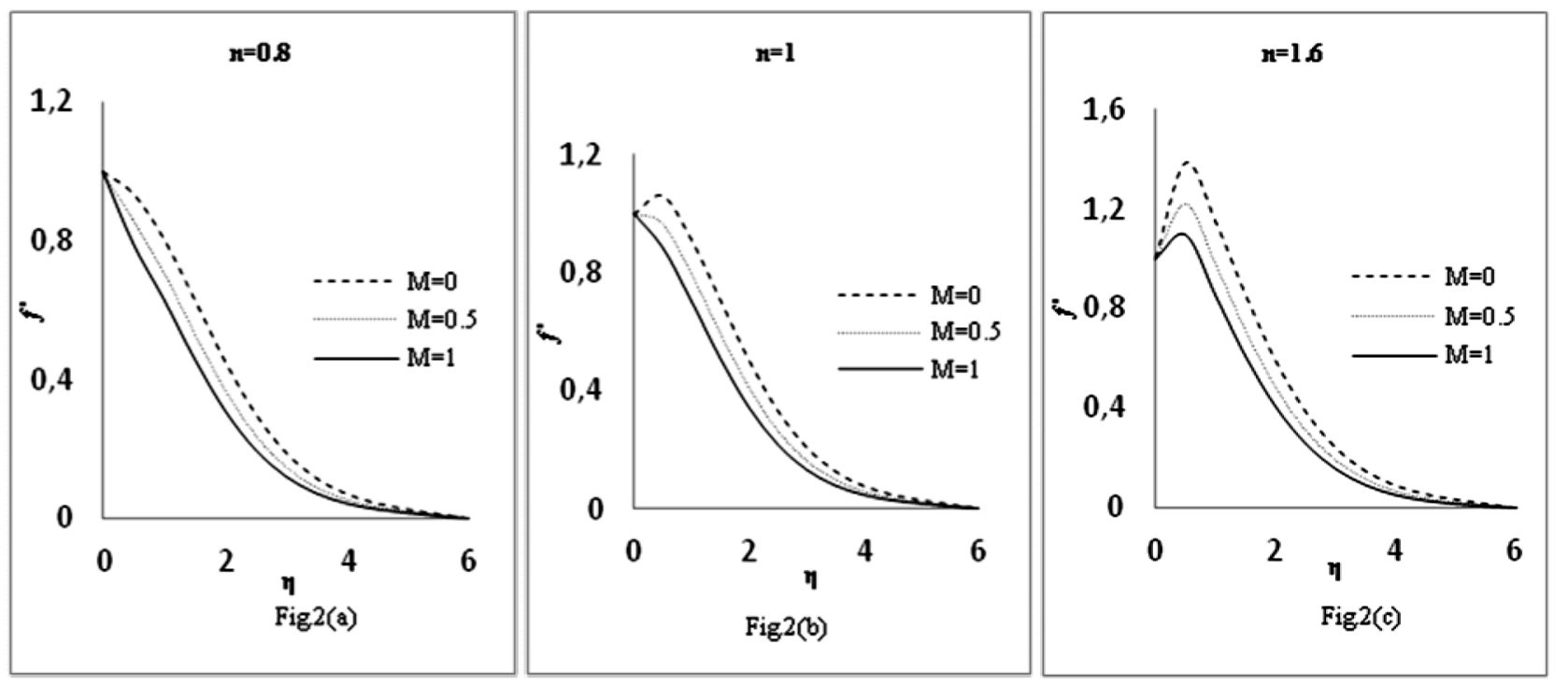

Fig.2. Velocity profiles for different values of $M$ for $m=0.1, \operatorname{Pr}=1, r=0, S=0, \mathrm{Ec}=0$ a) pseudo plastic fluids $(n=0.8)$ b) Newtonian fluids $(n=1)$ c) dilatent fluids $(n=1.6)$.
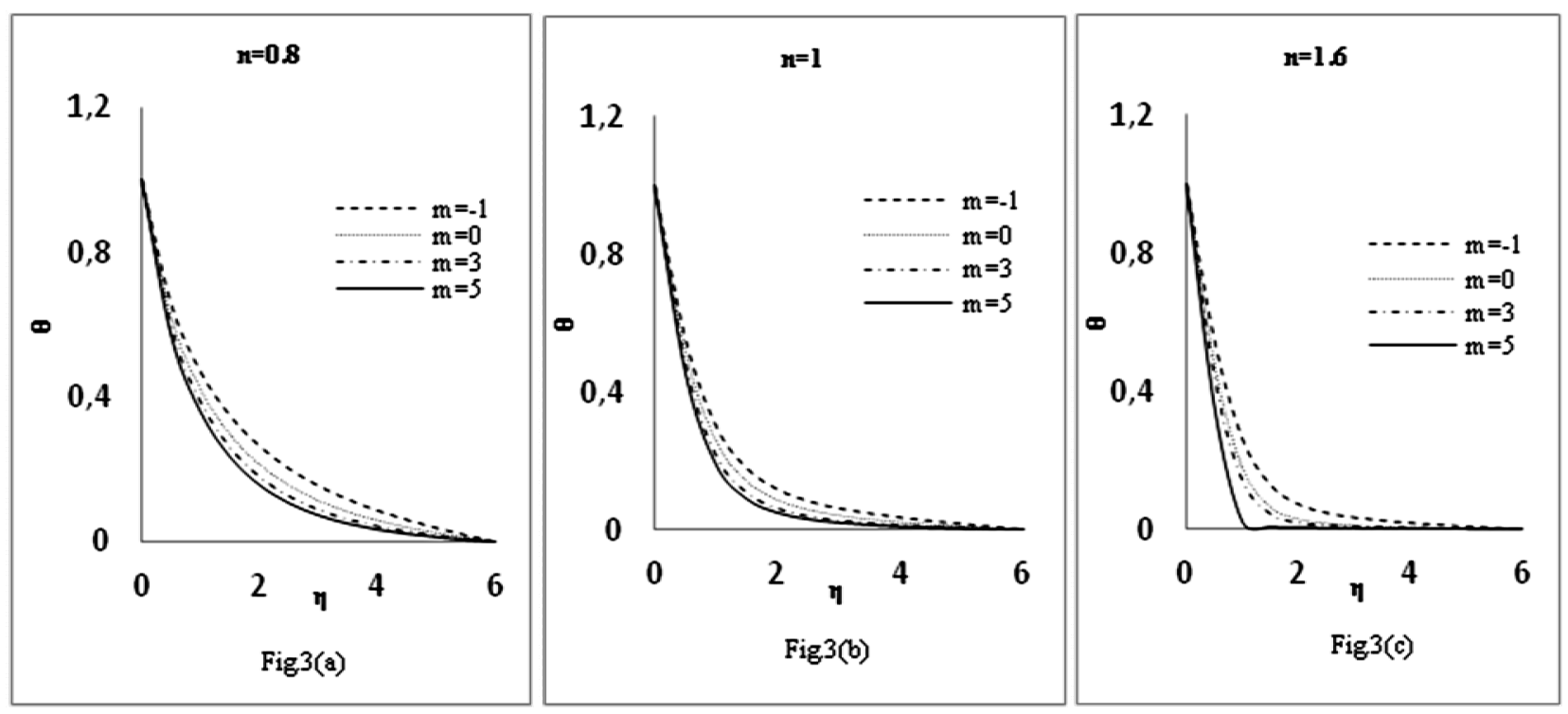

Fig.3. Temperature profiles for different values of $m$ for $M=0, \operatorname{Pr}=1, r=0, S=0, \mathrm{Ec}=0$ a) pseudo plastic fluids $(n=0.8)$ b) Newtonian fluids $(n=1)$ c) dilatent fluids $(n=1.6)$. 

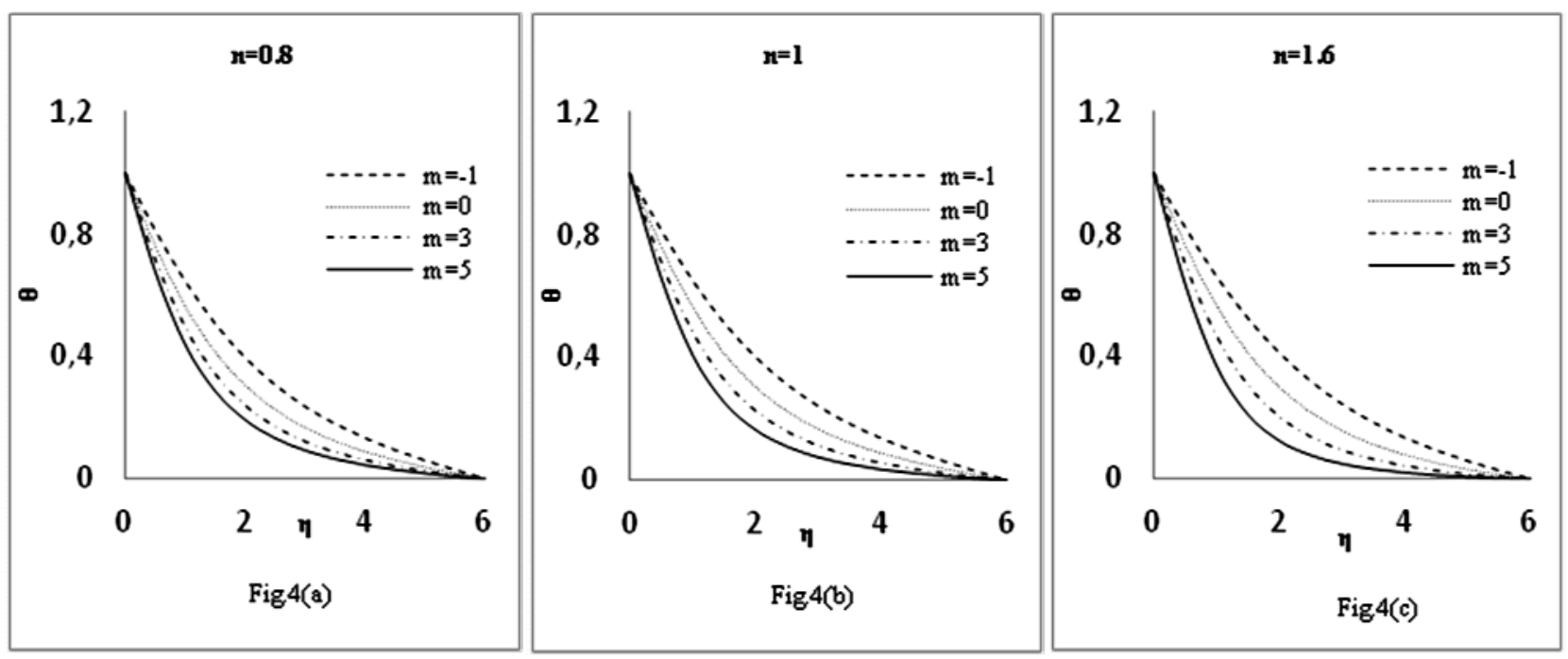

Fig.4. Temperature profiles for different values of $m$ for $M=1, \operatorname{Pr}=1, r=0, S=0, \mathrm{Ec}=0$ a) pseudo plastic fluids $(n=0.8)$ b) Newtonian fluids $(n=1)$ c) dilatent fluids $(n=1.6)$.

Figures 5 and 6 illustrate the dimensionless temperature profiles for different values of $m$ for the temperature exponent parameter $r=0.1$ and -0.1 , respectively. It follows from these figures that the effect of the stretching sheet parameter $m$ leads to a decrease in the temperature profiles. It can be seen that the effect is smaller when $r=0.1$ in comparison to its effect when $r=-0.1$. Figures $7 \mathrm{a}-\mathrm{c}$ illustrate the dimensionless temperature for different values of the modified Prandtl number $\operatorname{Pr}$ for a) pseudo plastic fluids $(n<1)$ b) Newtonian fluids $(n=1)$ c) dilatent fluids $(n>1)$. The temperature of the fluid decreases with the increase of the Prandtl number Pr. The effect is larger in pseudo plastic fluids in comparison to its effect in dilatent fluids. This is because of the fact that the boundary layer thickness decreases with an increase in the Prandtl number.
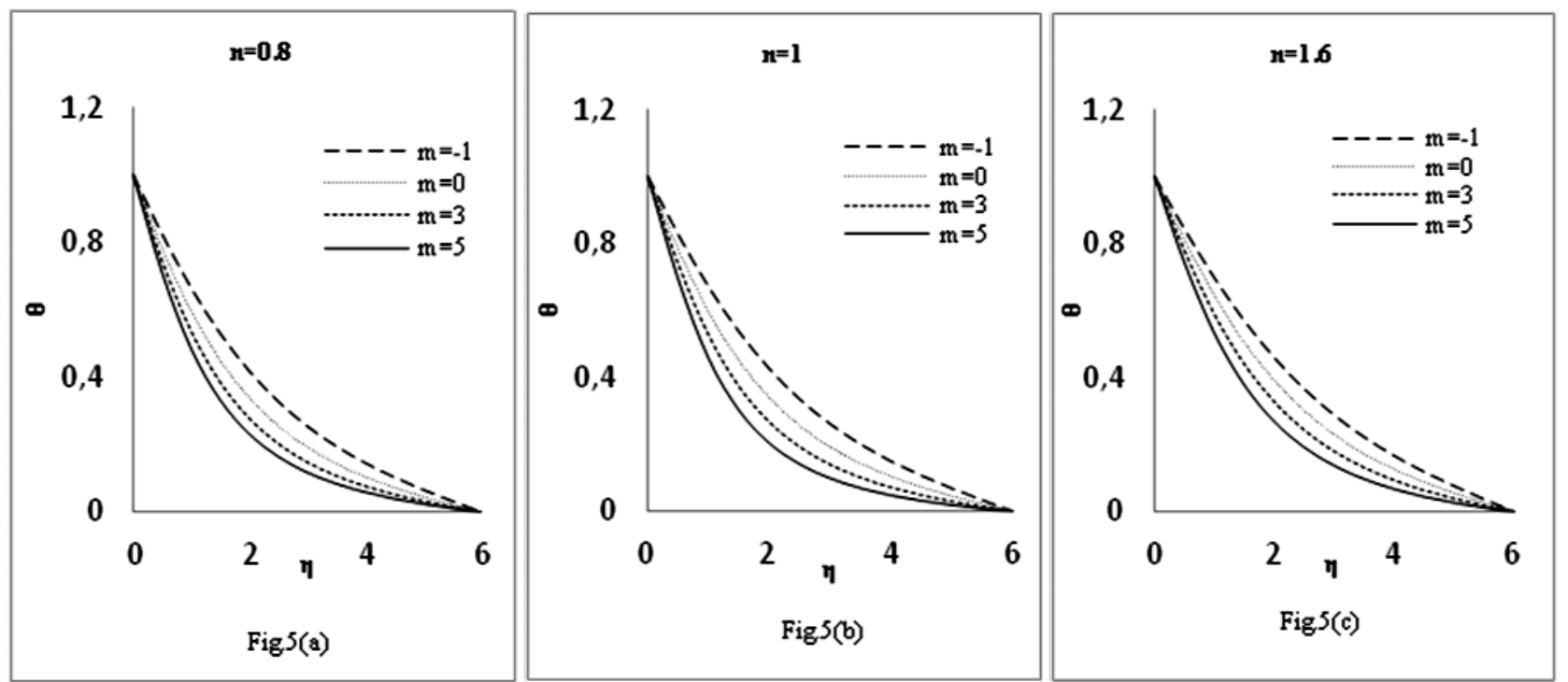

Fig.5. Temperature profiles for different values of $m$ for $M=1, \operatorname{Pr}=1, r=0.1, S=0, \mathrm{Ec}=0$ a) pseudo plastic fluids $(n=0.8)$ b) Newtonian fluids $(n=1)$ c) dilatent fluids $(n=1.6)$. 

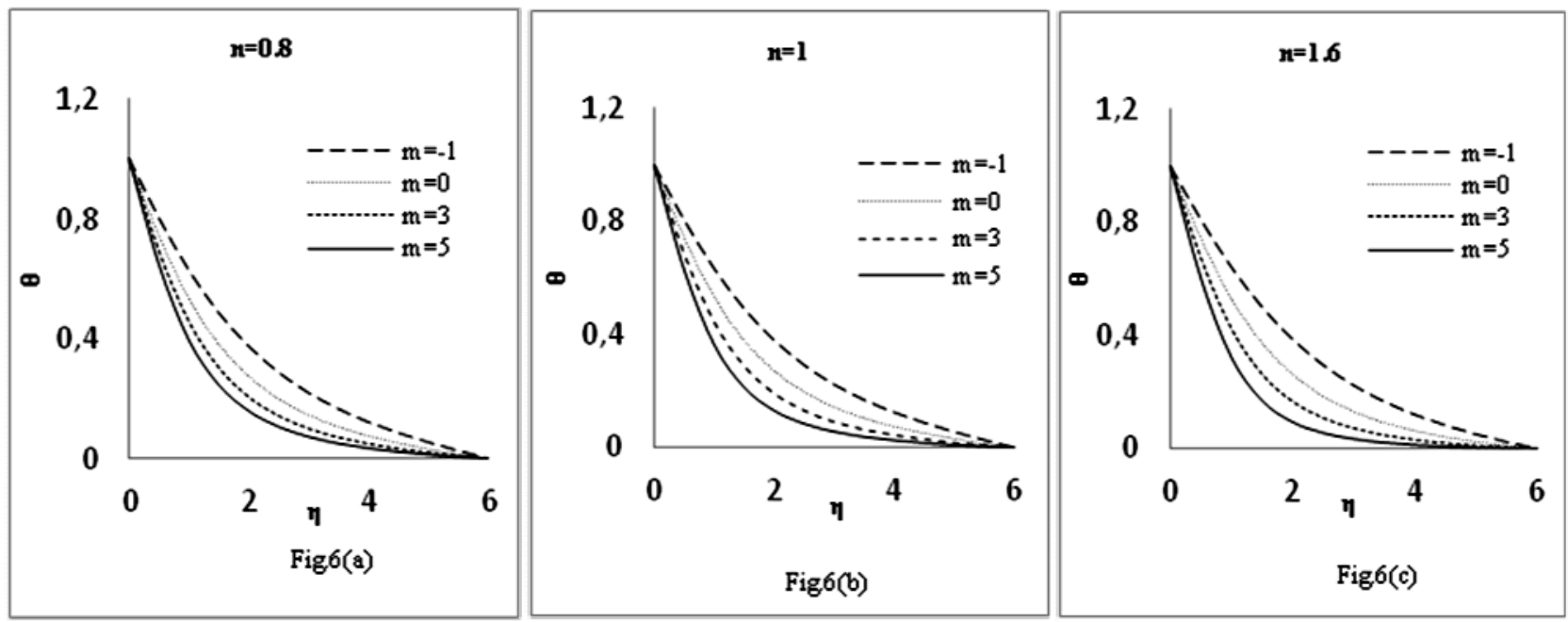

Fig.6. Temperature profiles for different values of $m$ for $M=1, \operatorname{Pr}=1, r=-0.1, S=0, \mathrm{Ec}=0$ a) pseudo plastic fluids $(n=0.8)$ b) Newtonian fluids $(n=1)$ c) dilatent fluids $(n=1.6)$.
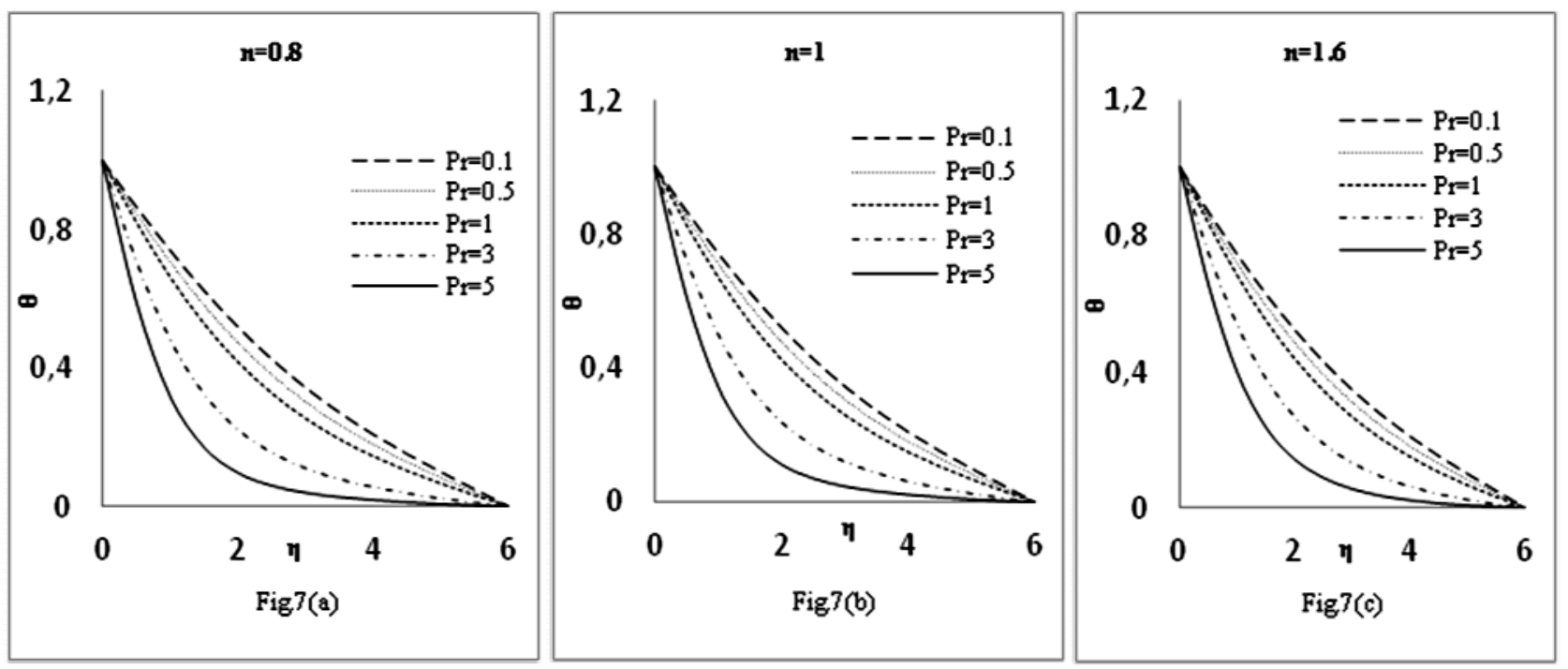

Fig.7. Temperature profiles for different values of $\operatorname{Pr}$ for $M=1, m=0.1, r=0.1, S=0, \mathrm{Ec}=0$ a) pseudo plastic fluids $(n=0.8)$ b) Newtonian fluids $(n=1)$ c) dilatent fluids $(n=1.6)$.

Figures 8a-c represent the dimensionless temperature profiles for different values of the Eckert number Ec for a) pseudo plastic fluids $(n<1)$ b) Newtonian fluids $(n=1)$ c) dilatent fluids $(n>1)$, respectively. It is observed that the dimensionless temperature profile $\theta$ increases with an increase of the Eckert number Ec. The effect of viscous dissipation is greater in pseudo plastic fluids in comparison with other fluids. In Figures 9a-c the temperature profiles $\theta$ are plotted for different values of the heat source/sink parameter $S$ for a) pseudo plastic fluids $(n>1)$ b) Newtonian fluids $(n=1)$ c) dilatent fluids $(n>1)$, respectively. From these figures we see that the temperature distribution is lower throughout the boundary layer for negative values of heat sink and higher for positive values of $S$ heat source as compared with the temperature profiles in the absence of the heat source/sink parameter i.e., $S=0$. 

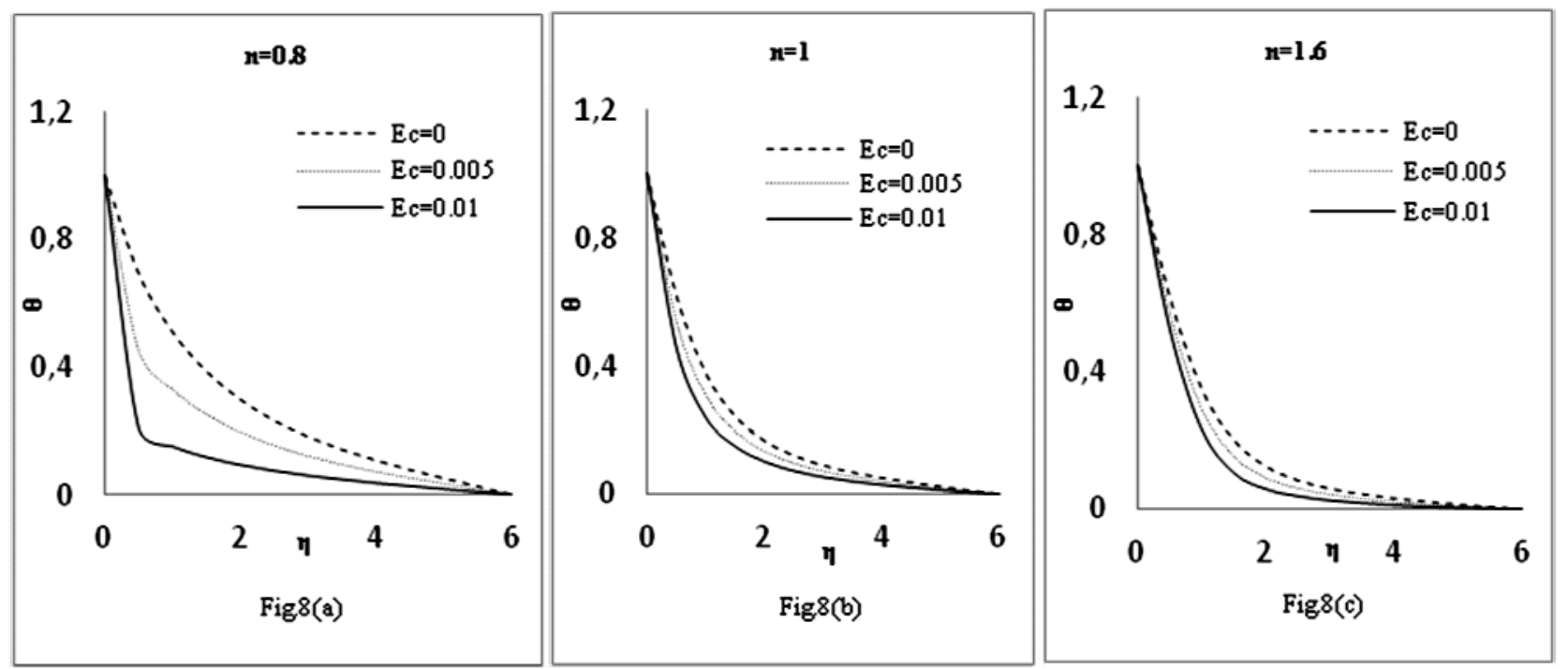

Fig.8. Temperature profiles for different values of Ec for $M=0, m=0.1, r=0.1, S=0, \operatorname{Pr}=1$ a) pseudo plastic fluids $(n=0.8)$ b) Newtonian fluids $(n=1)$ c) dilatent fluids $(n=1.6)$.
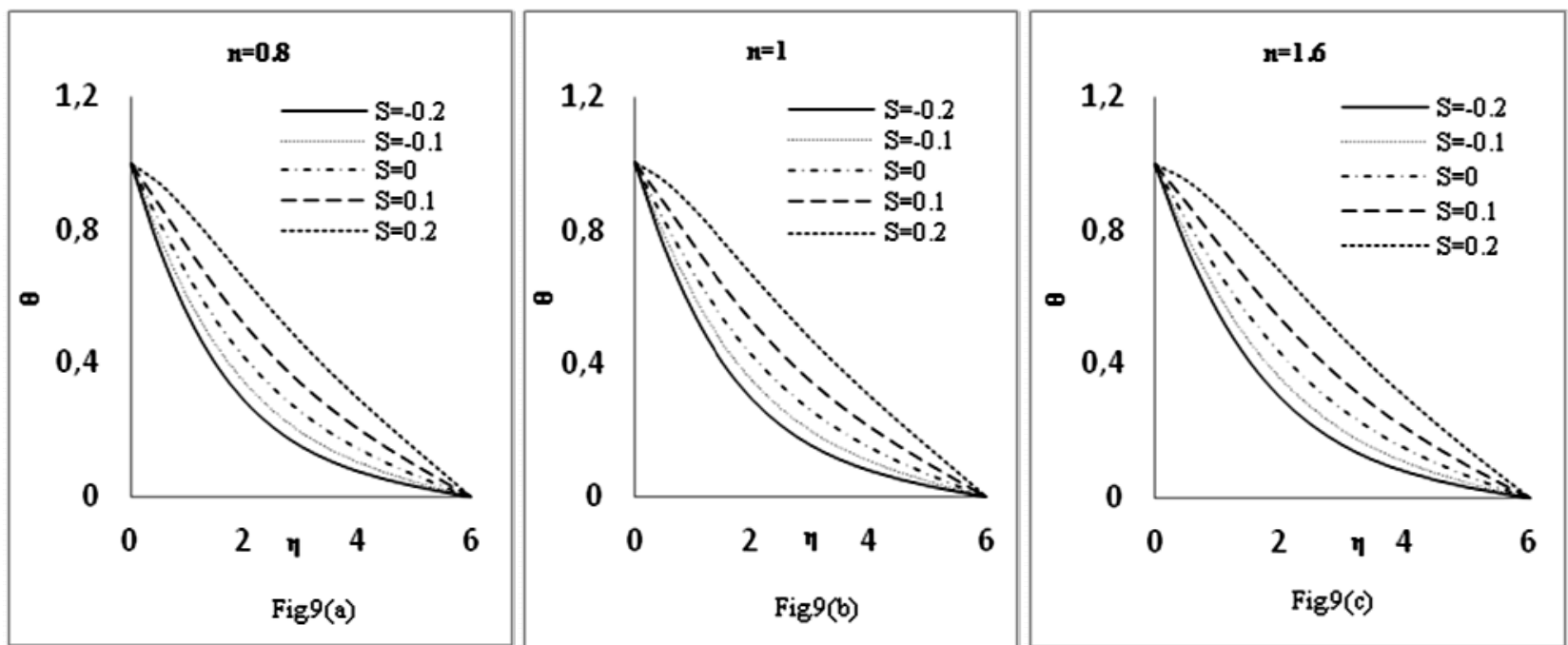

Fig.9. Temperature profiles for different values of $S$ for $M=1, m=0.1, r=0.1, \operatorname{Pr}=1, \mathrm{Ec}=0$ a) pseudo plastic fluids $(n=0.8)$ b) Newtonian fluids $(n=1)$ c) dilatent fluids $(n=1.6)$.

Figures 10a-c reveal the influence of the temperature exponent parameter $r$ for the fluid a) pseudo plastic fluids $(n<1)$ b) Newtonian fluids $(n=1)$ c) dilatent fluids $(n>1)$, respectively. The effect of increasing values of the temperature exponent parameter $r$ is to decrease the temperature profiles as noticed from the figures. The effects of the power law index $n$ on dimensionless velocity profiles $f^{\prime}$ and temperature profiles $\theta$ are plotted in Figs 11a and b, respectively. It can be seen from the figures that with an increase of the power law index $n$ the values of the velocity profiles $f^{\prime}$ increase. The dimensionless temperature profiles decrease with an increase of the power law index $n$ as noticed from Fig.11b. 

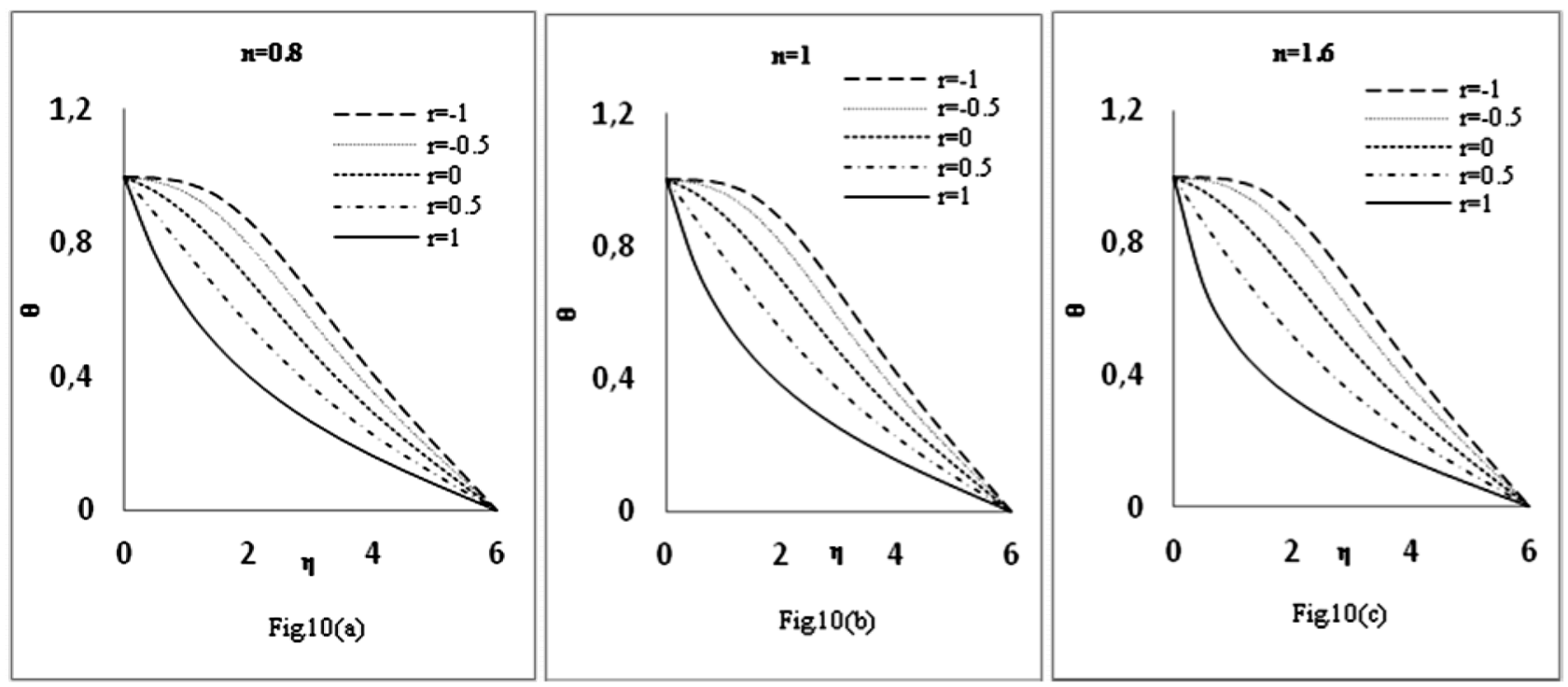

Fig.10. Temperature profiles for different values of $r$ for $M=1, m=0.1, S=0, \operatorname{Pr}=1, E c=0$ a) pseudo plastic fluids $(n=0.8)$ b) Newtonian fluids $(n=1)$ c) dilatent fluids $(n=1.6)$.

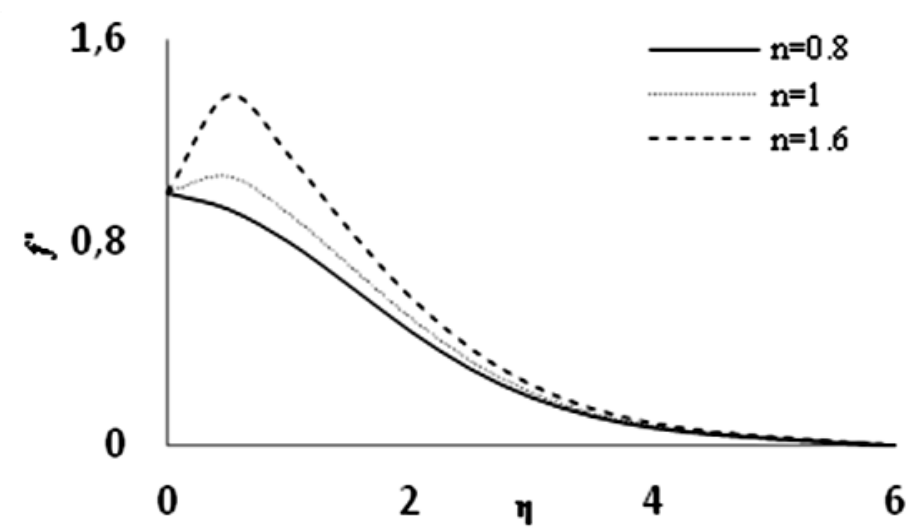

Fig.11a. Velocity profiles for different values of $n$ for $m=0.1, M=0, \operatorname{Pr}=1, r=0, S=0, \mathrm{Ec}=0$.

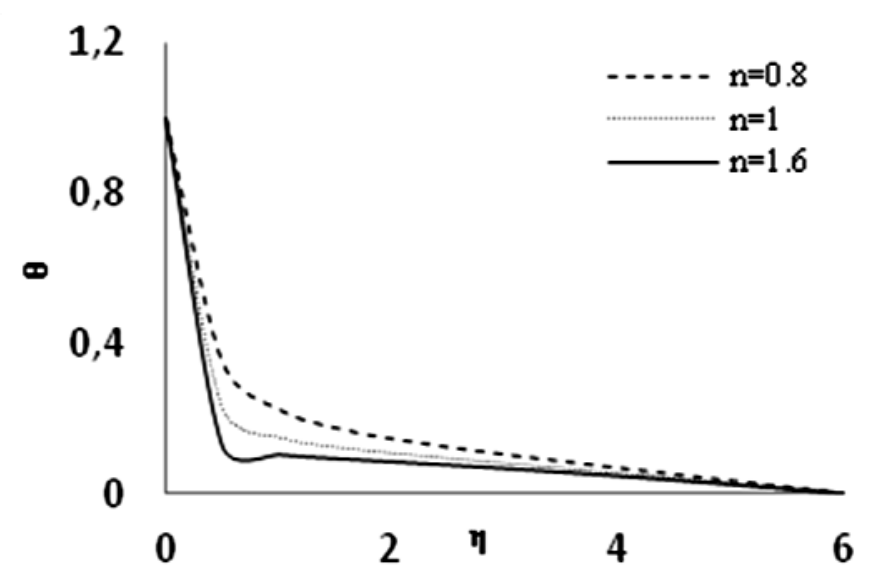

Fig.11b. Temperature profiles for different values of $n$ for $M=0.1, m=0.1, S=0, \operatorname{Pr}=5, \mathrm{Ec}=0, r=1$. 
Table1. Comparison of some of the values of the skin friction coefficient for $m=1$ for different values of $n$ and $M$.

\begin{tabular}{|l|l|l|l|}
\hline$n / M$ & 0.0 & 0.5 & 1 \\
\hline 0.8 & 1.1068 & 1.4646 & 1.7774 \\
\hline 1 & 1.0000 & 1.2247 & 1.4142 \\
\hline 1.6 & 0.979 & 1.1204 & 1.2389 \\
\hline
\end{tabular}

The values of the local skin friction coefficient $\left(-f^{\prime \prime}(0)\right)$ are shown in Tab.1 for different values of the power-law index $n$ and magnetic field parameter $M$. It is observed that with an increase in the magnetic field parameter $M\left(-f^{\prime \prime}(0)\right)$ values increase. The effect of the power-law index $n$ for fixed values of the magnetic parameter reduces $\left(-f^{\prime \prime}(0)\right)$.

Table 2. Numerical values of the Nusslet number for different values of physical parameters.

\begin{tabular}{|l|l|l|l|l|l|l|l|l|l|l|l|l|}
\hline \multicolumn{2}{|l|}{$r=-1$} & \multicolumn{1}{l|}{$r=0$} \\
\hline$S$ & $\operatorname{Pr}$ & $M$ & $m / r$ & $n=0.8$ & $n=1$ & $n=1.6$ & $n=0.8$ & $n=1$ & $n=1.6$ & $n=0.8$ & $n=1$ & $n=1.6$ \\
\hline & & & -0.3 & 0.36912 & 0.45484 & 0.41274 & -0.31214 & -0.27212 & -0.27753 & -0.77311 & -0.75516 & -0.75403 \\
\hline & & & 0 & 0.28616 & 0.32606 & 0.28208 & -0.34956 & -0.32934 & -0.34124 & -0.79451 & -0.78701 & -0.79141 \\
\hline 0 & 1 & 1 & 0.5 & 0.16291 & 0.14254 & 0.08196 & -0.40972 & -0.42086 & -0.44921 & -0.82981 & -0.84105 & -0.85801 \\
\hline & & & 1 & 0.05589 & -0.0081 & -0.06401 & -0.46671 & -0.50546 & -0.53794 & -0.86479 & -0.89441 & -0.91731 \\
\hline$m$ & $\operatorname{Pr}$ & $M$ & $S$ & $n=0.8$ & $n=1$ & $n=1.6$ & $n=0.8$ & $n=1$ & $n=1.6$ & $n=0.8$ & $n=1$ & $n=1.6$ \\
\hline & & & -0.1 & 0.14331 & -0.00841 & -0.10994 & -0.50891 & -0.57006 & -0.61511 & -0.95031 & -0.98301 & -1.00646 \\
\hline 1 & 1 & 0 & 0 & -0.04069 & -0.15634 & -0.23651 & -0.60546 & -0.65959 & -0.69924 & -1.01380 & -1.04544 & -1.06801 \\
\hline & & & 0.1 & -0.18012 & -0.27481 & -0.34241 & -0.68778 & -0.73702 & -0.77330 & -1.07135 & -1.10206 & -1.12404 \\
\hline
\end{tabular}

The numerical values of the Nusslet number for different values of physical parameters are shown in Tab.2. It is observed from the table that the effect of increasing values of the power-law index $n$ is to decrease the wall temperature gradient whereas a reverse trend is seen for the magnetic field parameter $M$ when $S=0$. The increasing values of the temperature exponent parameter $r$ and velocity exponent parameter $m$ reduce the values of $\left(-\theta^{\prime}(0)\right)$. This result has significance in industrial applications to reduce expenditure on power supply. The effects of the Prandtl number and heat source/sink decrease the wall temperature gradient.

\section{Conclusions}

In summary, the present study describes the power law fluid flow and heat transfer over a nonlinearly stretching sheet in the presence of a transverse magnetic field by taking into account viscous dissipation effects and heat source/sink parameter. From the numerical results and above discussions it can be found that

1) The effect of $m$ is to decrease the temperature profile $f^{\prime}$ and profile $\theta$ a) pseudo plastic fluids $(n=0.8)$

b) Newtonian fluids $(n=1)$ c) dilatent fluids $(n=1.6)$. 
2) The effect of increasing values of the magnetic parameter $M$ is to decelerate the velocity profile $f^{\prime}$.

3) The effect of the temperature exponent parameter $r$ and modified Prandtl number is to decrease the temperature profiles $\theta$.

4) The viscous dissipation effect is to increase the temperature profiles.

5) The heat source/sink parameter reduces the temperature profiles for $S<0$ (sink) and increase the temperature profiles for $S>0$ (source) when compared with $S=0$.

6) The effect of the power-law index parameter $n$ is to increase the velocity profile $f^{\prime}$ and reduces the temperature profiles $\theta$.

\section{Nomenclature}

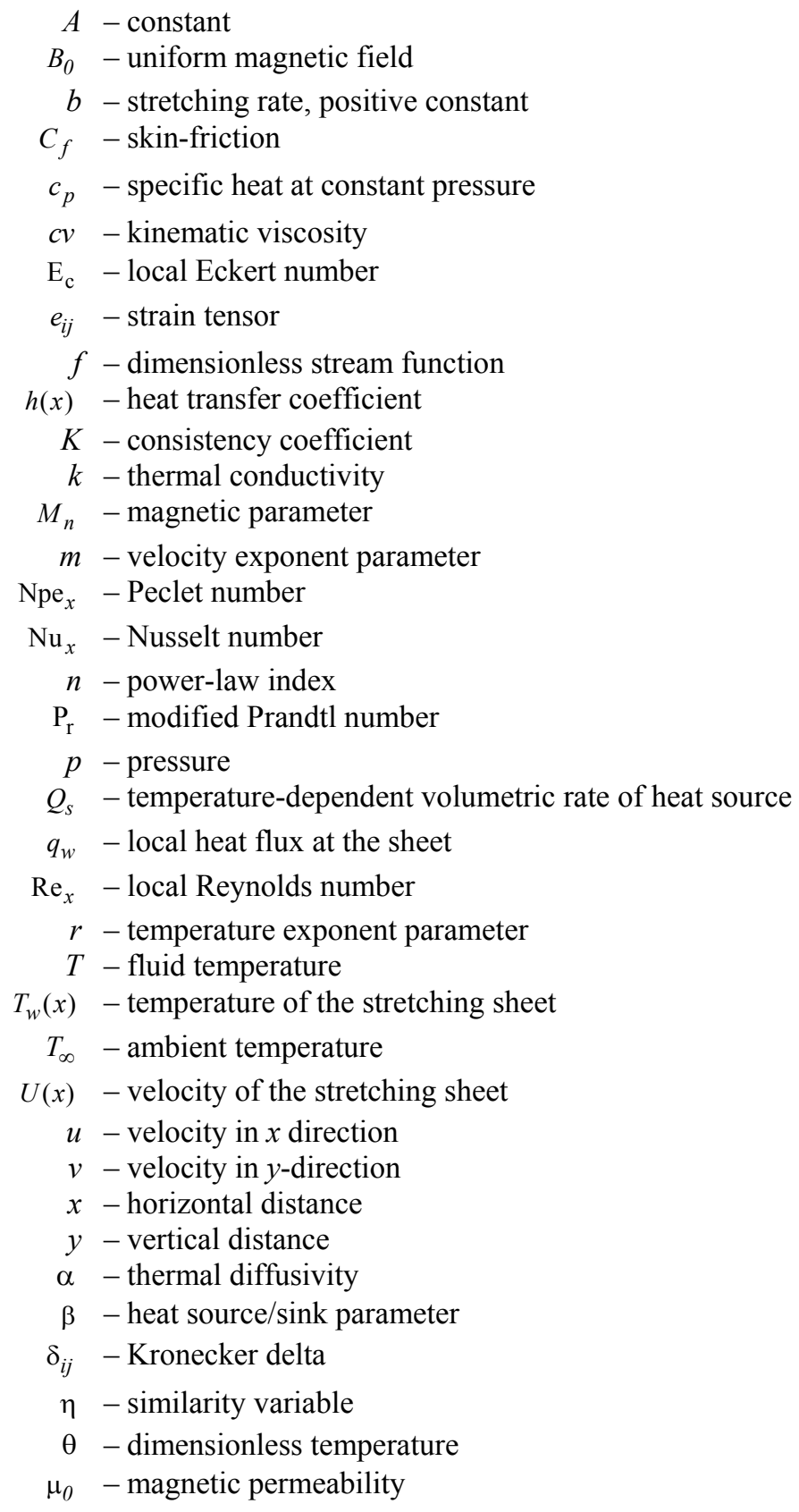




$$
\begin{aligned}
\rho & - \text { density } \\
\sigma & - \text { electrical conductivity } \\
\tau_{x y} & - \text { shear stress } \\
\psi & - \text { stream function }
\end{aligned}
$$

\section{Subscripts}

$w$ - condition at the stretching sheet

$\infty-$ condition at infinity

\section{Superscript}

' - differentiation with respect to $\eta$

\section{References}

Acrivos A., Shah M.J. and Petersen E.E. (1960): Momentum and heat transfer in laminar boundary layer flows of nonNewtonian fluids past external surfaces. - AICHE J., vol.6, pp.312-317.

Andersson H.I., Bech K.H. and Dandapat B.S. (1992): Magneto hydrodynamic flow of a power-law fluid over a stretching sheet. - International Journal of Non-Linear Mechanics, vol.27, No.6, pp.929-936.

Anjali Devi S.P. and Thiyagarajan M. (2006): Steady nonlinear hydro magnetic flow and heat transfer over a stretching surface of variable temperature. - Heat and Mass Transfer, vol.42, No.8, pp.671-677.

Bellman R.E. and Kalaba R.E. (1965): Quasi-Linearization and Non-Linear Boundary Value Problem. - New York: Elsevier.

Chaim T.C. (1995): Hydro-magnetic flow over a surface with a power-law velocity. - International Journal of Engineering Science, vol.33, No.3, pp.429-435.

Char M.I. (1994): Heat and mass transfer in a hydro-magnetic flow of a visco-elastic fluid over a stretching sheet. Journal of Mathematical Analysis and Applications, vol.186, No.3, pp.674-689.

Chen C.H. (2003): Heat transfer $n$ power-law fluid film over a unsteady stretching sheet. - Heat Mass Transfer, vol.39, pp.791-796.

Cortell R. (2005): A note on magneto hydrodynamic flow of a power-law fluid over a stretching sheeet. - Applied Mathematics and Computation, vol.168, No.1, pp.557- 566.

Crane L.J. (1970): Flow past a stretching plate. - Zeitschrift für Angewandte Mathematik und Physik, vol.21, No.4, pp.645-647.

Denier J.P. and Dąbrowski P.P. (2004): On the boundary layer equations for power-law fluids - Proceedings of the Royal Society of London, vol.A, pp.3143-3158.

Emad M., Abo-Eldajab and Salem A.M. (2005): MHD free-convective flow of a non-Newtonian power-law fluid at a stretching surface with a uniform free-stream. - Heat Mass Tranfer, vol.169, pp.806-818.

Gupta P.S. and Gupta A.S. (1977): Heat and mass transfer on a stretching sheet with suction or blowing. - The Canadian Journal of Chemical Engineering, vol.55, No.6, pp.744-746.

Ishak A., Nazar R. and Pop I. (2006): Magneto hydrodynamic stagnation-point flow towards a stretching vertical sheet. - Magneto Hydrodynamics, vol.42, pp.17-30.

Jadhav J.P. and Waghmode B.B. (1990): Heat transfer to non-Newtonian power-law fluid past a continuously moving porous flat plate with heat flux. - Heat and Mass Transfer, vol.25, No.6, pp.377-380.

Mahmoud M.A.A. and Mahmoud M.A.E. (2006): Analytical solution of hydro magnetic boundary-layer flow of a nonNewtonian power-law fluid past a continuously moving surface. - Acta. Mech., vol.18, pp 183-89. 
Rajagopal K.R., Gupta A.S. and Na T.Y. (1983): A note on Falker-skan flows of a non-Newtonian fluid. - International Journal of Non-Linear Mechanics, vol.18, pp.313-319.

Sakiadis B.C. (1961): Boundary-layer behavior on continuous solid surfaces: Boundary-layer equations for twodimensional and axis symmetric flow. - AICHE J., vol.7, pp.26-28.

Sarpakaya T. (1961): Flow on non-Newtonian fluids in a magnetic field. - AIChE Journal, vol.7, pp.26-28.

Schowalter W.R. (1960): The application of boundary layer theory to power-law pseudo plastic fluid similar solutions. - AICHE J., vol.6, pp.24-28.

Zheng L.C., Ma L. and He J. (2000): Bifurcation solution to a boundary layer problems arising theory of power law fluids. - Acta Math. Sci., vol.20, pp.19-26.

Received: September 23, 2013

Revised: October 10, 2013 